\title{
OBESITY \\ microRNA-dependent control of MODY gene expression contributes to obesity-associated insulin resistance
}

The transcription factor HNF-1 $\beta$ - a deficiency of which is known to cause a rare form of maturity-onset diabetes mellitus of the young (MODY)- has been implicated in the development of obesity-associated insulin resistance.

"Genetic variation and posttranslational modification of components of the insulin signalling pathway cannot fully explain the clinical association of obesity and insulin resistance," explains senior investigator Jens C. Brüning (Max-Planck-Institute for Neurological Research, Cologne, Germany). Hence, his team investigated the contribution of post-transcriptional gene silencing via microRNAs (miRNAs) to the development of obesity-associated insulin resistance. "We aimed to achieve a high coverage in detecting miRNAs whose expression is altered in the liver of obese mouse models through microarray technology," says Brüning.

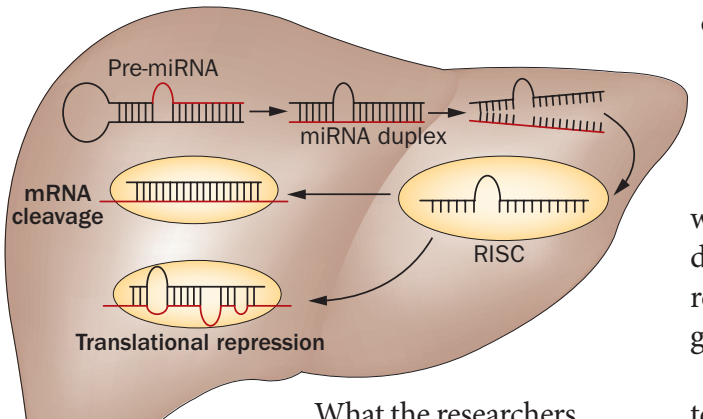

diabetes variant MODY 5, which is also known as RCAD (renal cysts and diabetes syndrome). "Knockdown of $H N F 1 B$ expression in the mouse liver using small hairpin RNA caused insulin resistance, which, to our knowledge, is the first demonstration of a role for HNF- $1 \beta$ in the regulation of hepatic insulin sensitivity and glucose metabolism," comments Brüning. In the future, the investigators hope to test the feasibility of miR-802 antagomirschemically engineered oligonucleotides that would prevent miR-802 from binding to the HNF1B mRNA molecule and thus preclude silencing-to treat obesity-associated insulin resistance and type 2 diabetes mellitus in nonhuman primate studies.

\section{Linda Koch}

miR-802 expression in obese mice improved glucose metabolism. Brüning and co-workers then identified the target of miR-802-dependent silencing: HNF1B. Mutations in this gene cause the rare genetic
Original article Kornfeld, J.-W. et al. Obesity-induced overexpression of miR-802 impairs glucose metabolism through silencing of Hnf1b. Nature 494, 111-115 (2013) 